\title{
VHF Partial Discharge Detection during After-Laying testing of Power Cables
}

\author{
S. Meijer ${ }^{1 *}$, R.A. Jongen ${ }^{1}$, E. Gulski ${ }^{1}$, P.P. Seitz ${ }^{2}$, T.J.W.H. Hermans ${ }^{3}$ and L. Lamballais ${ }^{3}$ \\ ${ }^{1}$ High-voltage Components and Power Systems, Delft University of Technology, \\ PO Box 5031, 2600 GA Delft, Netherlands \\ ${ }^{2}$ Seitz Instrum ents AG, CH-5443 Niederrohrdorf, Switzerland \\ ${ }^{3}$ Prysmian Cables and Systems B.V., P.O. Box 495, 2600 AL Delft, Netherlands \\ *E-mail : s.meijer@tudelft.nl
}

\begin{abstract}
In this paper the results of after-laying testing of transmission power cable accessories using a nonconventional technique are being presented. The nonconventional system is based on the detection of high frequency signals being emitted by the partial discharge. Therefore, each power cable accessory is equipped with either an internal or external sensor to decouple the partial discharge signals. The partial discharge detection system uses narrow-band detector, in this case a spectrum analyzer.
\end{abstract}

This technique however is very difficult to calibrate due to different factors affecting the frequency response of the measuring setup. This paper also presents a sensitivity check which can be used to validate the sensitivity of the system for a certain required PD level. Examples of on-site sensitivity verification are presented, before the actual PD measurements take place. An example of this system is shown taken from several measurements performed in the field.

\section{INTRODUCTION}

Partial discharges (PD) can occur in medium and high voltage cable systems $[1,2]$. PD is a localized dielectric breakdown of a small portion of a solid or liquid electrical insulation system under high voltage stress. A common way to test a new power cable with accompanying accessories is rather uncomplicated. For example, the Dutch AC-test requirement for routine testing a $150 \mathrm{kV}$ power cable according to the NEN 3630 standard [3] is applying $220 \mathrm{kV}(2.5 \times \mathrm{U} 0=2.5 \times$ $150 \mathrm{kV} / \sqrt{3}$ ) for 10 minutes. The tests are considered successful if the cable and its accessories did not break down.

Nowadays, detection of partial discharge activity is one of the most sensitive means to detect insulation deficiencies inside high voltage equipment [4]. Insulation deficiencies are the origin of partial discharge activity which precedes a complete break down [1]. So, the size of partial discharges are giving an indication of the quality of the installation of transmission power cable accessories and detection of these discharges is becoming an important issue during the on-site acceptance test. The system is verified for the presence of partial discharge activity while raising the applied high voltage in small steps. If partial discharges seem to appear, the test is immediately abandoned and a complete breakdown is prevented.

Accessories like cable joints and -terminations, are the most vulnerable parts of a cable setup, because of the electric field enhancement at the edge of truncated conductors and dielectrics. Furthermore, discharging defects can originate during installation of the cable onsite: poor workmanship and entrapment of dust or water particles due to lack of cleanliness can evolve to partial discharges that will increase with time and eventually lead to failure of the cable system.

Therefore, to ensure the quality of installed power cable accessories, partial discharge detection during the onsite acceptance test is more and more becoming an important issue. Occurrence of PD activity can be used to assess the condition of the cable accessory and proper action can be taken. The set-up of a PD detector, operating in the very high frequency (VHF) or ultra high frequency (UHF) range is shown in figure 1.

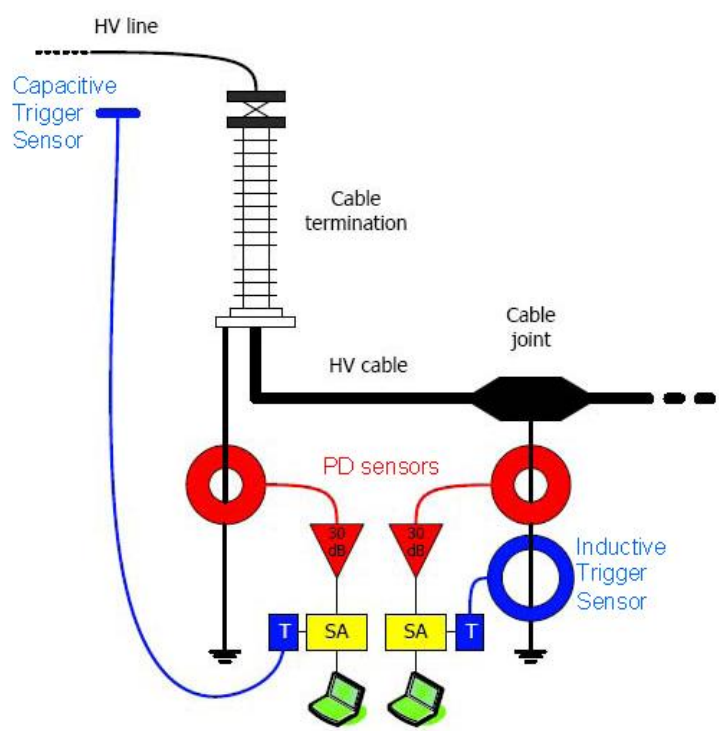

Figure 1: Two VHF/UHF PD detection system as installed in the field consisting of internal inductive sensors around the earth connection, a coaxial cable, a $30 \mathrm{~dB}$ VHF/UHF pre-amplifier, a spectrum analyzer, a trigger sensor and PC. 


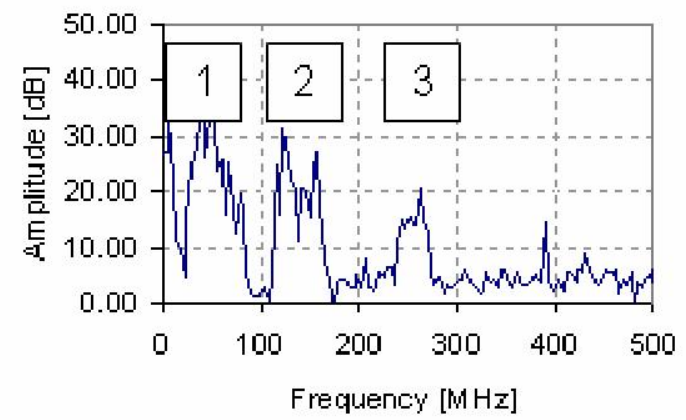

Figure 2: Example of a signal-to-noise ratio frequency spectrum as has been measured on-site. Here three frequency ranges show additional activity which could originate from partial discharges.

\section{VHF/UHF PD DETECTION}

The VHF/UHF partial discharge detection method is based on the fact that partial discharges are fast transient phenomena. In case of partial discharge phenomena in power cables, $\mathrm{PD}$ pulses with frequencies of up to 500 $\mathrm{MHz}$ have been detected. In case of an after-laying test of power cables, PD detection in higher frequency bands is advantageous for two reasons: less influence of external noise and selective testing of only the accessories.

The first point is also well-known from measurements taken in the field of GIS and transformers [5]. The first step in making a measurement is capturing the frequency spectrum. Based on this spectrum, a certain centre frequency which represents $\mathrm{PD}$ activity with the highest signal-to-noise ratio is selected, as indicated in figure 2. Secondly, a spectrum analyser can also analyse the coupler's signals in the time domain, resulting in similar phase-resolved PD patterns that are obtained with a standardized measuring circuit. Such phaseresolved $\mathrm{PD}$ patterns offers the possibility to recognize a certain type of defect and to discriminate between insulation defect and noise.

Secondly, the method is rather discriminating because at higher measuring frequencies, it can only detect PD

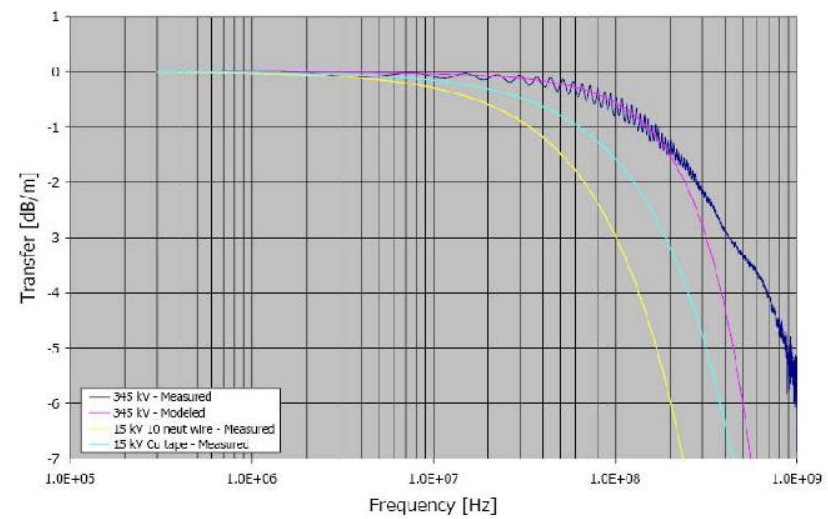

Figure 3: Simulated and measured HF attenuation of different XLPE insulated power cables activity in the accessory itself or in a small part of the cable very close to the accessory. This is due to the fact that above $100 \mathrm{MHz}$, the damping of the high frequency signals is high, see figure 3 (the thinner the cable, the higher the damping). This is an advantage, because for new installed power cables, PD activity is expected to occur only in the accessories, because these are installed in the field and during the installation some defects might have been introduced. The power cable itself can be assumed to be PD-free as it is tested in the factory for any $\mathrm{PD}$ activity.

So if any $\mathrm{PD}$ activity is observed during the afterinstallation test of a new power cable system, and the detection frequency is at frequencies above $100 \mathrm{MHz}$, it can be concluded that there is a defect in the cable accessory the PD detection system is connected to.

\section{LABORATORY TEST}

Using a narrow-band $\mathrm{VHF} / \mathrm{UHF}$ technique, the first step is measuring the full frequency spectrum $F 1$ up to 500 $\mathrm{MHz}$ using a sweeptime of $500 \mathrm{~ms}$. Secondly, a frequency spectrum $F 2$ is measured using a sweeptime of 5 seconds. As a result, both spectra will show the continuous noise signals present, however, if there is any $\mathrm{PD}$ activity, more pulses will be captured during the 5 seconds sweep compared to the 500 millisecond sweep. So by subtracting spectrum F1 from F2, a signalto-noise ratio is obtained. The peaks inside this SNRspectrum might indicate the presence of partial discharge activity, see figure 2 .

Secondly, a certain peak is selected and as the spectrum analyser can also analyse the coupler's signals in the time domain, similar phase-resolved PD patterns that are obtained with a standardized measuring circuit. Such phase-resolved $\mathrm{PD}$ patterns offers the possibility to recognize a certain type of defect and to discriminate between insulation defect and noise. An example of an obtained PD pattern for an artificial cavity in a cable joint in a laboratory set-up is shown in figure 4 .

By this result the applicability of the technique was demonstrated and the next step is to determine the sensitivity for insulation defects that are still detectable. For that purpose, a sensitivity check procedure has been defined and will be described in the following section.

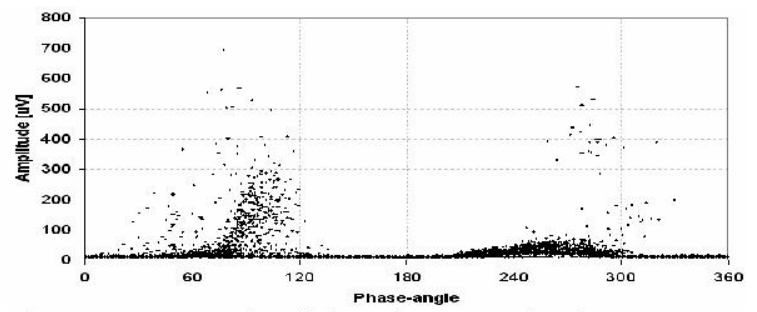

Figure 4: Example of the phase resolved PD pattern for an small cavity in a cable joint. 


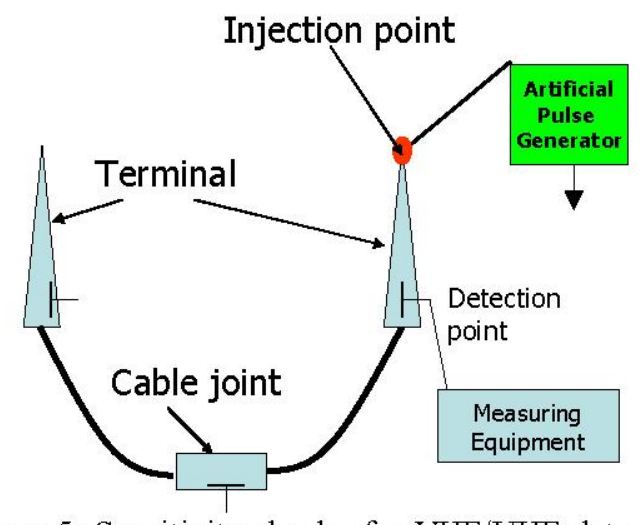

Figure 5: Sensitivity check of a VHF/UHF detection circuit for on-site PD detection on power cables.

\section{SENSITIVITY CHECK PROCEDURE}

When performing partial discharge measurements, the calibration of the detection system is an important issue. For PD detection systems according to the IEC 60270 standard, this calibration procedure is well defined and described in the standard. However, such a calibration procedure is at present not possible nor available for VHF/UHF PD detection systems. In particular, the highfrequency behavior of the sensor, the type and routing of the measurement cables, the selected measuring frequency and measuring bandwidth have a strong influence on the magnitude of the detected signals by the detection equipment used during the PD measurements.

Therefore, to determine the response of the detection circuit on PD pulses, a sensitivity check was performed. The basic circuit of this sensitivity check is shown in figure 5. A similar but slightly adapted sensitivity check procedure as is available for UHF PD detection on gasinsulated systems should consist of the following steps [6]:

1) In a laboratory setup use a PD-source with known magnitude $(\mathrm{pC})$ and measure the frequency spectrum.

2) Use an artificial pulse generator to inject pulses into the termination and change the amplitude until a frequency spectrum is detected which is comparable to the measured frequency spectrum originating from the

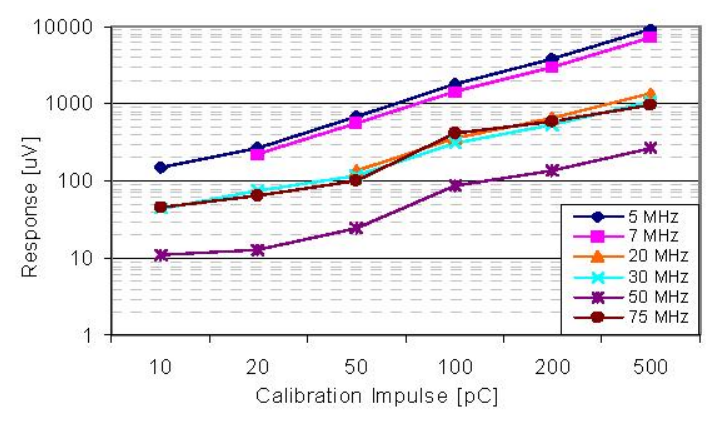

Figure 6: Example of an on-site sensitivity check: response of the $\mathrm{VHF} / \mathrm{UHF}$ detection system to calibrator pulses at different measuring frequencies.

PD source (see step 1). In this way, the frequency response of the detection circuit at this termination can be determined.

From this test, the relation between the calibrated PD magnitude of a certain real PD source and artificial pulse is known for a certain measuring circuit. For 380 $\mathrm{kV}$ power cables it was shown that an artificial pulse of $1 \mathrm{~V}$ represents $10 \mathrm{pC}$ of PD magnitude [4]. Now, injection of these voltages can be used to test the sensitivity of the on-site measuring circuit by the step 3 . 3) Sensitivity check of the on-site measuring circuit by injecting the pulse shape found under step 2 on each termination. If the injected signal is detected, this means that the sensitivity is high enough to detect partial discharge activity with a certain magnitude in $\mathrm{pC}$ as defined under step 1. A result of such an on-site sensitivity check is shown in figure 6 .

\section{FIELD APPLICATION}

Above mentioned system was being applied during acceptance tests of new high-voltage power cables. One example is described below. Figure 7 shows the lay-out of the cable system. In fact, an existing three-phase 110 $\mathrm{kV}$ oil-filled cable with one reserve phase has been split to install a GIS substation. The connection between the GIS and the existing cable system is made of new XLPE cables. During the after-laying test, PD activity is monitored at the XLPE-oil transition joints (figure 8) and the GIS terminations.

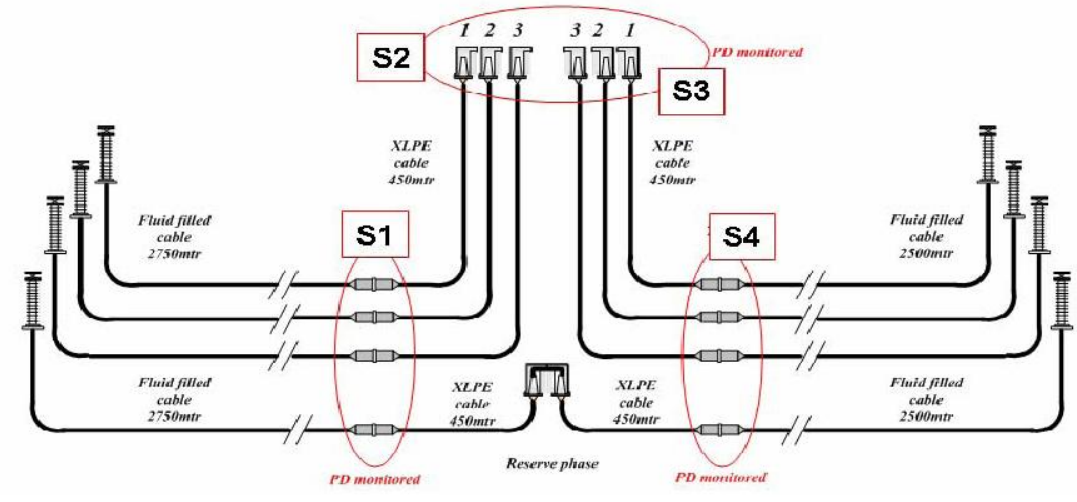

Figure 7: Lay-out of the cable system under test.

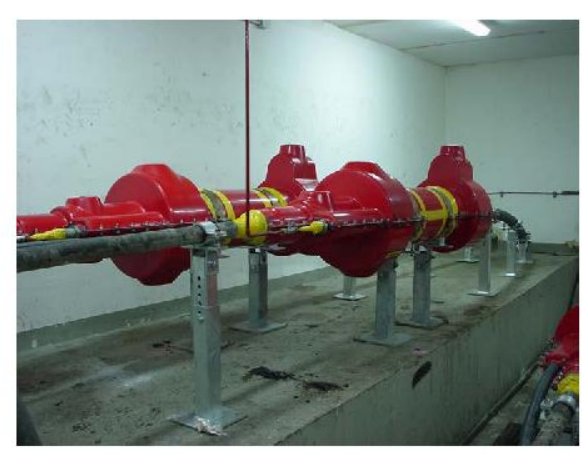

Figure 8: Oil-XLPE cable transition joint 


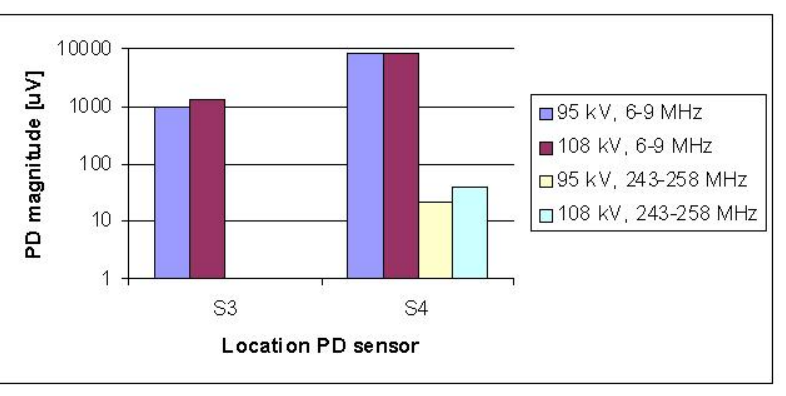

Figure 9: Example of measuring results obtained in phase 3 .

Figure 9 shows detected PD levels in [uV] as obtained at two sensor locations in phase 2. The PD pattern shows similarities with internal discharges as can be seen in figure 10 .

Furthermore, the following observations can be made from figure 9. First of all, the PD levels detected in a low frequency range of $6-9 \mathrm{MHz}$ at sensor $\mathrm{S} 4$ are higher than at sensor S3. Secondly, in a higher frequency range of 243-258 MHz, PD activity was only detected at sensor S4. Therefore it can be concluded that the discharge activity is closer to sensor S4 than sensor S3.

Table 1: Typical damping for a high-voltage power cable in different frequency ranges.

\begin{tabular}{ll}
\hline Frequency range $[\mathrm{MHz}]$ & Damping $[\mathrm{dB} / \mathrm{km}]$ \\
\hline $6-9 \mathrm{MHz}$ & $31.3 \mathrm{~dB} / \mathrm{km}$ \\
$243-258 \mathrm{MHz}$ & $4000 \mathrm{~dB} / \mathrm{km}$ \\
\hline
\end{tabular}

Moreover, as described before, the damping of the cable depends on the frequency, see figure 3 . The attenuation of this $110 \mathrm{kV}$ cable for the two used frequency ranges is shown in table 1. Applying the values mentioned in table to the obtained PD magnitude of $8000 \mu \mathrm{V}$ at sensor S4, the detectable PD magnitude at sensor S3 can be estimated. In case of the low frequency range, the estimated PD magnitude is $1500 \mu \mathrm{V}$, which corresponds rather well with the detected $1300 \mu \mathrm{V}$. In case of the high frequency range, the attenuation is very high and no signals are expected to be detected on sensor S4 which is confirmed by the measurement

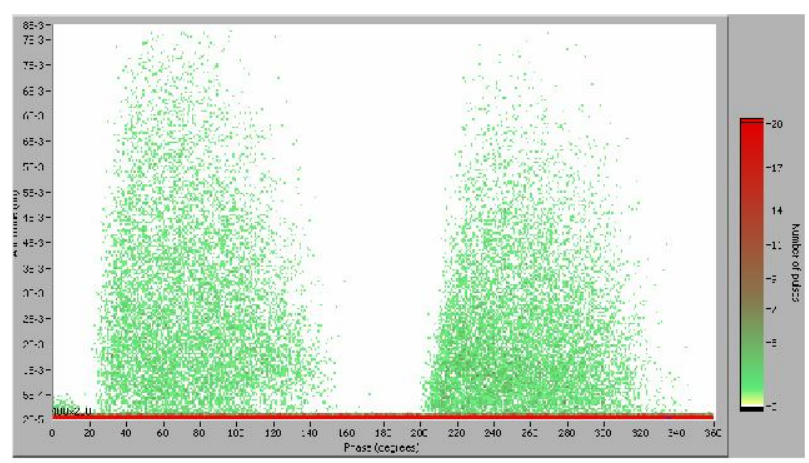

Figure 10: Example of a phase-resolved PD pattern as obtained in phase 3 .
From the determined frequency response during the sensitivity check the PD level was estimated to be 70 $\mathrm{pC}$. This level was stable during the 1 hour test and no breakdown occurred.

Based on these results it was concluded that there is PD activity in phase 3 . Due to the fact that in higher frequencies these PD's are not detectable at the GIS terminations (S3) it can be stated that the GIS terminations are PD-free. At the joint the PD activity was measured also at the higher frequencies. Therefore it can be stated that the PD activity has its origin near these joints, in the oil-filled part of the cable system. The utility decided to take the cable system into service, but have regular oil samples at the joints to monitor the development of the PD activity.

\section{CONCLUSIONS}

Based on the investigations described in this contribution, the following conclusions can be drawn concerning the VHF technique:

1. It is a flexible and non-intrusive technique suitable for $\mathrm{PD}$ detection during after-laying tests of power cables;

2. Sensitive enough to detect $\mathrm{PD}$ of $10 \mathrm{pC}$ in cable terminations by selecting a frequency range with good signal-to-noise ratio;

3. PD site can be estimated by comparison of data obtained in low- resp. high-frequency ranges;

4. Calibration procedure needs further development in order to ensure reproducible calibration and be more representative of PDs.

\section{REFERENCES}

[1] F.H. Kreuger, "Industrial High Voltage I," DUP, Delft, The Netherlands, 1992.

[2] F.H. Kreuger, "Industrial High Voltage II," DUP, Delft, The Netherlands, 1992.

[3] NEN 3630:2004 en. "Cables with insulation of XLPE and their accessories for voltages of $50 \mathrm{kV}$ up to and including $150 \mathrm{kV}$," ICS code 29.060.20, Commission number 364020, 2004.

[4] S. Meijer, et. al., "Simultaneous Condition Assessment of Accessories of Power Cables using a Wireless VHF/UHF PD Detection System" in $\mathrm{CMD}$ conference proceedings, Changwon, Korea, 2006.

[5] B.F. Hampton, R.J. Meats, "Diagnostic measurement at UHF in GIS," IEE Proceedings Vol. 135, Pt. C. No.2, pp. 137-144, March 1988.

[6] CIGRE TF 15/33.03.05, "PD Detection System for GIS: Sensitivity Verification for the UHF Method and the Acoustic Method", Electra No.183, April 1999. 